\title{
Research on Microstructure and Hardness of Bimetal Composite Layer Between LAS and HCCI
}

\author{
Wenbing ZHANG ${ }^{1}$, Shumei $\mathrm{CHEN}^{2}$, Hui GAO${ }^{1}$, Yongchang $\mathrm{ZHU}^{3 *}$ \\ ${ }^{1}$ School of Mechanical and Electronic Engineering, Beijing Polytechnic College, Beijing, China \\ ${ }^{2}$ School of Mechanical Engineering, Jiamusi University, Jiamusi, China \\ ${ }^{3}$ School of Materials science and Engineering, Jiamusi University, Jiamusi, China \\ e-mail: zwb8788@163.com,csm1964@163.com,gh19962727@163.com, *jmsdxzyc@163.com
}

\begin{abstract}
Based on the casting technique analysis on the temperature field and the velocity field for the low alloy steel, low alloy steel(LAS)/ high chromium white cast iron(HCCI) bimetal wear steel plate was prepared by two liquid bimetal composite casting process. The microstructure of interface layer of wear-resisting plate was studied. A clear and distinguishable composite layer between the LAS and the HCCI was detected with the SEM. The composite layer from the LAS to the HCCI can be divided into pearlite transition layer, composite layer and HCCI transition layer. The Vickers hardness from the LAS layer to the HCCI layer was $360 \mathrm{HV}$ to $960 \mathrm{HV}$. There are some granular carbides in the longitudinal growth dendritic austenite of the HCCI transition layer. According to the principle of the solute redistribution at the solid-liquid interface, the morphology of the original austenite presented the distinct directional fir-tree crystal growth in accordance with planar, cellular and dendritic.
\end{abstract}

Keywords-two liquid casting; wear-resisting plate; composite layer; dendritic austenite

\section{INTRODUCTION}

Bimetal composite wear steel plate is widely used in the fields of metallurgy, cement, electricity and mining industries under corrosive wear, impact abrasion and frictional wear. By means of multilayer and multi pass lap surfacing, the wear surfacing layer with big thickness and area can be acquired on the substrate. The substrate of bimetal wear steel plate is usually low carbon steel, low alloy steel or stainless steel. These materials own more reliable toughness for supporting various load and impact[1-3]. High hardness wear materials for wear layer are used to resist to different wear conditions. Currently, automatic welding technology was mainly used to produce the bimetal wear steel plate, and the self-shield alloy wire was soldered to the low carbon steel or low alloy steel plate surface. The building-up welding composite layers were welded together during the non-uniform heating process, resulting in the residual stresses. So this way may cause a lot of marked lateral cracks. The base metal cladding layer was diluted, resulting in the uneven performance of the surfacing layer from the top and the bottom. Thus overall wear resistant layer was insufficient[4-6]. With the progress of welding technology and equipment, some related technologies were not widely applied due to the high cost of the corresponding method and immature technology.

Two-liquid casting composite process can realize large metallurgical composite plane between different metals. These mentioned soldering defects existing in building-up welding composite layers can be avoided. But using this composite process may lead to mixture, shrinkage and inclusions and other defects[7, 8]. So far, two-liquid bimetal plate products mainly focused on the application of the liner, jaw and back plate, etc. al. So using two-liquid bimetal casting wear plate to replace the building-up welding wear plate is still blank. Therefore, to study two liquid bimetal composite casting large flat plate is significant $[9,10]$.

Two-liquid casting process of bimetal wear plate based on the comprehensive needs of working conditions, and mechanical properties of the substrate and wear layer can easily adjust the chemical contents of the HCCI wear layer and the LAS substrate[11, 12]. The process ensured the excellent metallurgical quality of wear layer and effectively reduced the substrate alloy composition dilution effect on the wear layer, so that a good metallurgical bond between the HCCI and the LAS was achieved. The full metallurgical bond may ensure the wear layer without any crack.

\section{EXPERIMENTAL METHODS}

\section{A. Experimental Materials}

Two medium frequency furnaces were equipped for smelting the HCCI and LAS whose compositions were listed in Table 1. The $\mathrm{CO}_{2}$ blowing harden water-glass sand was chosen as the moulding material, and the design dimension of the wear plate was $520 \mathrm{~mm} \times 400 \mathrm{~mm} \times 40 \mathrm{~mm}$.

TABLE I. TWO KINDS OF ALLOY CHEMICAL CONTENTS (WT. \%)

\begin{tabular}{|c|c|c|c|c|}
\hline Contents & $\mathbf{C}$ & $\mathbf{S i}$ & $\mathbf{M n}$ & $\mathbf{C r}$ \\
\hline LAS & $0.3 \sim 0.5$ & $0.8 \sim 1.0$ & $1.5 \sim 2.0$ & $0.8 \sim 1.6$ \\
\hline HCCI & $3.1 \sim 3.2$ & $0.2 \sim 0.4$ & $0.5 \sim 0.7$ & $22 \sim 25$ \\
\hline
\end{tabular}

\section{B. Experimental Preparation}

Based on double gating system of compound casting technology, the composite casting process was designed in figure 1. The LAS and HCCI pouring basins were used for the pouring and feeding, and HCCI riser was mainly used to observe the flow of LAS and to feed the molten HCCI. The wear plate mold cavity should be given to an inverse deformation design in advance according to the casting size. The total area of the steel plates as the external chilling 
materials at the bottom of the cavity central region was $520 \mathrm{~mm} \times 400 \mathrm{~mm}$ in dimension, and then the thickness of the plates was about $0.5 \mathrm{~T}$ (where $\mathrm{T}$ is the diameter of the hot spot of the LAS, equals to the thickness of the LAS)[13]. Laying chilling materials were to accelerate cooling the base material and increase the temperature gradient, and thus the temperature field was controlled. The poured temperature of LAS and HCCI should be controlled in $1500^{\circ} \mathrm{C} \sim 1550^{\circ} \mathrm{C}$, and the time interval between LAS and HCCI was 5 20s. After the liquid LAS poured into the mold cavity, the HCCI was casting the second pouring system which the temperature gradient was larger in the vertical direction, but the horizontal temperature was uniform.

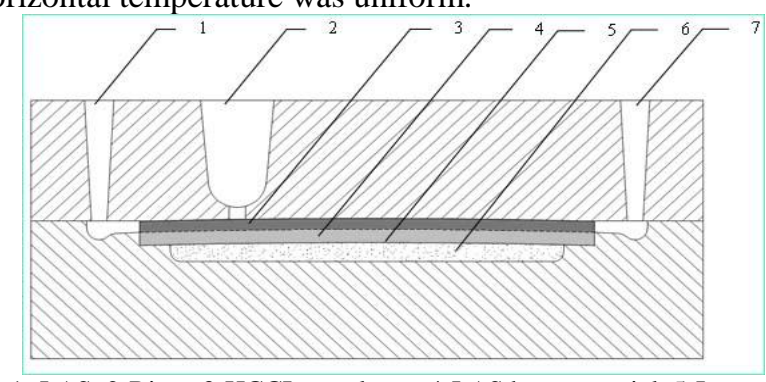

1- LAS, 2-Riser, 3-HCCI wear layer, 4-LAS base material, 5-Inverse deformation, 6-Chilling materials, 7-HCCI pouring basin

Figure 1. Two-liquid casting wear plate composite process.

\section{Experiment Equipments}

Two medium frequency furnaces were used for smelting LAS and HCCI respectively. The temperature field was detected by the Sixteen Channel Tour-inspector. The microstructure was observed by the OLYMPUS-GX71 Electronic Microscope and JSM-6360LV Scanning Electron Microscope. The Vickers hardness of compound layer and transition layer was measured by the microhardness instrument.

\section{TWO-LIQUID BIMETAL COMPOSITE PROCESS ANALYSIS}

\section{A. Influence of the Metal Fuidity on the Composite Layer}

There is a greater longitudinal temperature gradient in the LAS under the action of the chilling material. When the lower part had solidified, the upper surface still remained a small amount of liquid or solid - liquid mixed state. Under gravity casting conditions, the flow pattern of liquid metal along the gating system was turbulent when it was poured into the sodium silicate sand mold cavity flow, resulting in the HCCI direct flushing to LAS. The LAS surface appeared temporarily slight oxide and a small amount of slag was carried along with the liquid LAS. Under the HCCI flow scouring, the oxide and slag were conveniently detached from the contact region between the two kinds of metal, and then liquid connection between the HCCI and LAS ensured a metallurgical compound layer of the large plane. In the process of LAS casting, the velocity of the front molten steel was larger, but there was no fluid splash phenomenon so that the iron beans or cold insulation defects were prevented. After the molten LAS rising from the bottom of the mold cavity, the superficial liquid metal was tending towards stability. The flow velocity of the metal liquid on top tends to smooth fluctuations, but that of the metal liquid near the bottom is to the follow-up composite technology without adverse effects. The late poured liquid HCCI is connected with the LAS surface liquid. Under the action of the intense heat of HCCI, LAS presented a thin remelting layer and began to solidify upward to the HCCI, so the HCCI/LAS bimetal composite layer was formed. The bimetal composite casting plate was prepared in Figure 2a. The section can be seen the distinct interface between LAS and HCCI in figure $2 \mathrm{~b}$.

\section{B. Effect of Temperature Field on the Composite Layer}

In order to realize the metallurgical bond between the LAS and HCCI, large gaps were left between the laid chilling materials on the bottom of the mold cavity, which was available for breathability. Through a practical casting between the HCCI and LAS, a full metallurgical bonding layer was realized. The HCCI infiltrated into the surface layer of LAS in a local region. As it was shown in Figure 3a, the sharp interface frontier state above the chilling materials existing larger gaps indicated that the infiltration temperature was higher, LAS liquid viscosity and surface tension were smaller, and then the foreign high temperature HCCI encountered the LAS causing the partial melting mixture. As LAS liquid viscosity and the surface tension were larger, the interface presenting a transition of cutting-edge arc above the smaller gaps of the chilling materials was seen in Figure $3 \mathrm{~b}$. The high temperatures HCCI was locally mixed with the melted LAS. The microstructure growth under the constitutional undercooling showed directional growth.
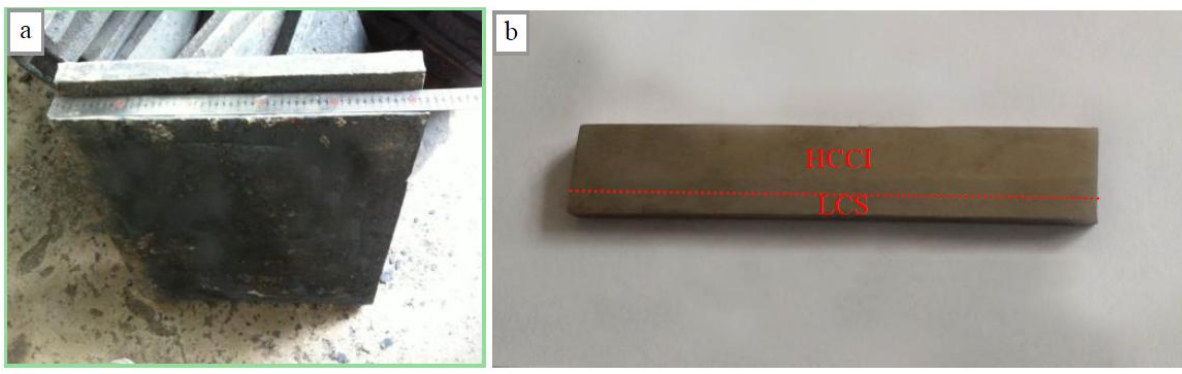

Figure 2. Two-liquid casting wear plate composite plate and sample:(a) Composite casting wear plate, (b) Longitudinal plane of the sample. 

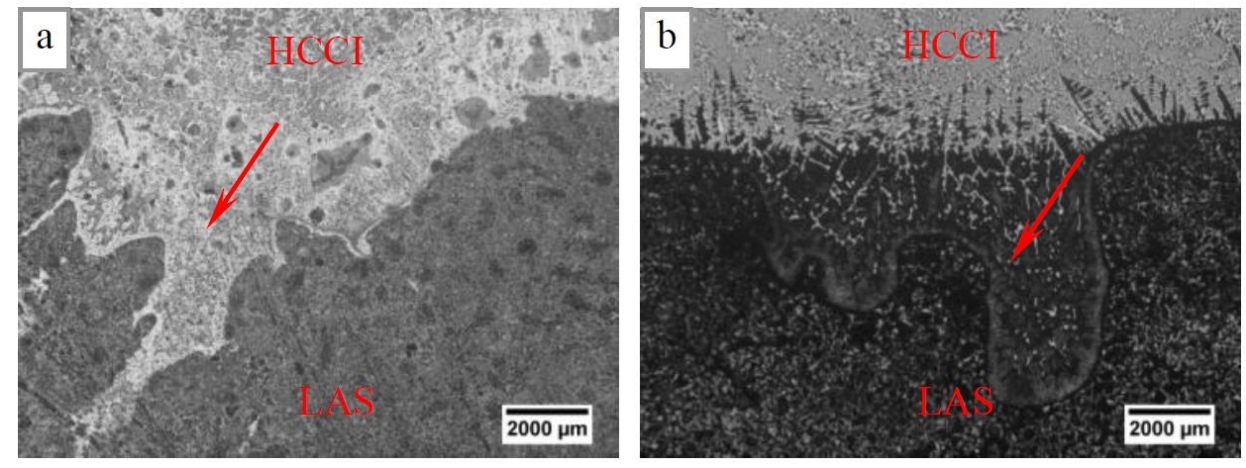

Figure 3. Microstructure of partial melting mixture at composite layer: (a) The sharp partial mixture at the interface frontier, (b) The cutting-edge arc partial mixture at the interface frontier.
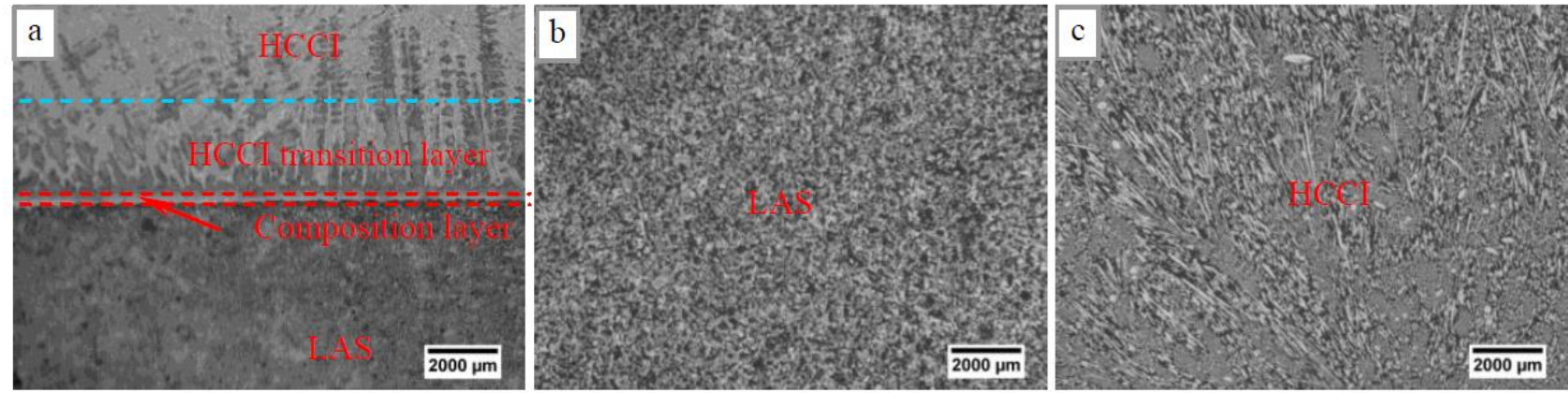

Figure 4. Two-liquid bimetal composite plate microstructure: (a) Composite layer and transition layer microstructure, (b) LAS and (c) HCCI.

\section{EXPERIMENTAL RESULTS AND DISCUSSION}

\section{A. Microstructure of Composite Layer}

The original hypereutectic HCCI composition gradient was divided into hypoeutectic, eutectic and hypereutectic eutectic near the composite layer and fusion transition layer. A small amount of eutectic transformation microstructure was mainly $\gamma-\mathrm{Fe}+\mathrm{M}_{7} \mathrm{C}_{3}$ among the longitudinal dendritic austenite, and the HCCI mircostructure above the dendritic austenite was the eutectic transformation products in Figure 4a. Liquid HCCI contacted with liquid LAS film surface, and then the original crystal nucleus or dendrite inside the original liquid film surface was remelted under the action of external heat. With the increasing of remelted region depth, the heat provided by the foreign liquid HCCI was not enough to remelt the LAS, so the heterogeneous nucleation began to occur on the LAS surface. The austenite nuclei below the composite layer first began to grow as planar crystal towards liquid HCCI interior, and the composite layer thickness was about $200 \mu \mathrm{m}$ based on the SEM. The solidification temperature gradient of the LAS can be controlled by the number and location of the chilled material. The LAS microstructure was mainly ferrite and pearlite in Figure 4b. The HCCI microstructure was mainly composed of primary eutectic carbide and eutectic ledeburite. From Figure 4c, the first eutectic carbides were mainly $(\mathrm{Fe}, \mathrm{C})_{7} \mathrm{C}_{3}$ which showed the hexagonal prismatic or hexagonal pyramid-shaped crystals. Hexagonal crystal section internally presented the visible filled metal holes of the austenite or austenitic transformation products. The remained liquid phase turned into the $\gamma-\mathrm{Fe}+(\mathrm{Fe}, \mathrm{C})_{7} \mathrm{C}_{3}$ eutectic, and $(\mathrm{Fe}, \mathrm{C})_{7} \mathrm{C}_{3}$ carbide showed the strip or bar form which presented a clear regularity in size. The carbide was apt to vertical surfaces and the increasing size of the microstructure.

The scanned composite layer and its vicinity were shown in Figure 5a. The sequence of the microstructure from the LAS to the HCCI was follows: LAS $(\alpha-\mathrm{Fe}+\mathrm{P}) \rightarrow$ pearlite transition layer $(\mathrm{P}) \rightarrow$ composite layer $(\gamma-\mathrm{Fe}+$ granular carbide) $\rightarrow$ HCCI layer (dendritic austenite $(\gamma-\mathrm{Fe}+$ granular carbide) + ledeburite among dendritic austenite $(\gamma-\mathrm{Fe}+$ $\left.\left.\mathrm{M}_{7} \mathrm{C}_{3}\right)\right) \rightarrow \mathrm{HCCI}$ (eutectic transformation ledeburite $(\gamma-\mathrm{Fe}+$ eutectic carbides $\left.\left(\mathrm{M}_{7} \mathrm{C}_{3}\right)\right) \rightarrow$ primary eutectic carbides $\left(\mathrm{M}_{7} \mathrm{C}_{3}\right)$ + eutectic transformation ledeburite $\left.\left(\gamma-\mathrm{Fe}+\mathrm{M}_{7} \mathrm{C}_{3}\right)\right)$, as was shown in figure $5 \mathrm{~b}$ and $5 \mathrm{c}$.

\section{B. Properties of Composite Layer}

According to Figure $6 a$ and $b$, the microhardness of the composite layer and transition layer showed regular changes, which provided an evidence for the good metallurgical bond. The Vickers hardness of pearlite transition layer was from 320 to 340 , and the Vickers hardness range of HCCI transition layer was from 940 to 960 . The Vickers hardness range of the composite layer was from 350 to 860 between the LAS transition layer and the HCCI transition layer. 

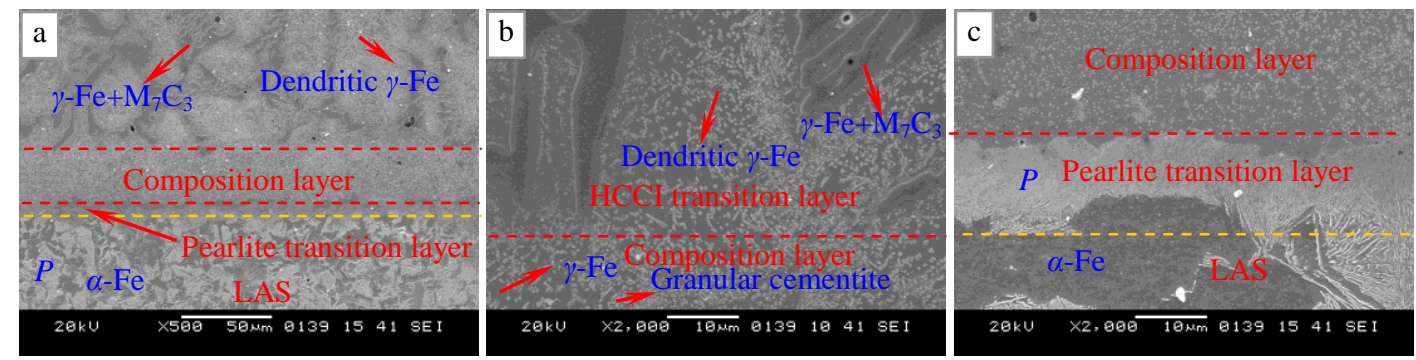

Figure 5. Two-liquid bimetal composite plate SEM microstructure: (a) LAS transition layer + Composite layer + HCCI transition layer, (b) Composite layer + HCCI transition layer, (c) Composite layer + LAS transition layer.
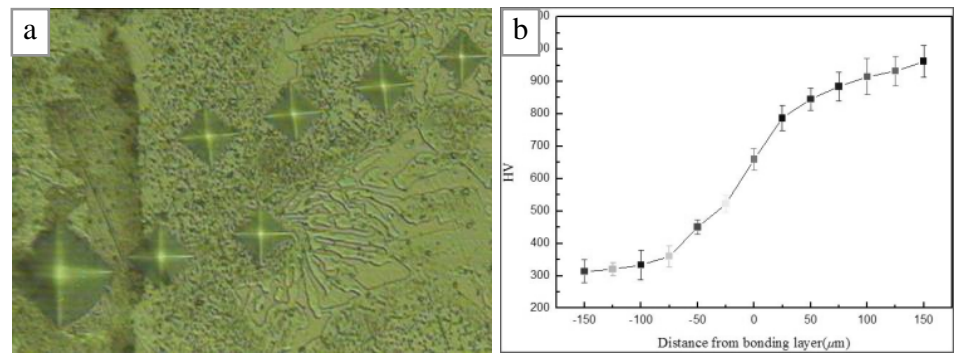

Figure 6. Composite layer and transition layer Vickers hardness analysis: (a) Vickers hardness analysis route, (b) Vickers hardness values.

\section{CONCLUSIONS}

(1) The LAS/HCCI wear-resisting plate about 40mm was prepared by the bimetal composite casting process, and the composite interface between the LAS and the HCCI realized the metallurgical bond.

(2) Under the action of chill materials, the austenite grew as dendrite between the composite layer and the HCCI transition layer under constitutional undercooling.

(3) With the constitutional undercooling region extension at the solidification in front of the liquid phase, the composite interface can been divided into six layers, and the Vickers hardness from the LAS layer to the HCCI layer was $360 \mathrm{HV}$ to $960 \mathrm{HV}$ gradually.

\section{ACKNOWLEDGMENT}

This work was financially supported by the Science Fund of Education Department of Beijing City, General Project No.KM201610853006, the Educational Department General Project of Heilongjiang Province under grant No. 2016KYYWF-0553.

\section{REFERENCES}

[1] X. F. Xiao, S. P. Ye, and W. X. Yin, "High Cr white cast iron/carbon steel bimetal liner by lost foam casting with liquid-liquid composite process," China Foundry, vol. 2, 2012, pp. 136-142.

[2] S. F. Rong, C. Liu, and J, W, Guo, "Influence of casting technique on the mechanical properties of bimetal composites," Journal of Iron and Steel Research International, vol. 31, 2012, pp. 562-565.

[3] S. F. Rong, H. T. Zhou, and Y. C. Zhu, "Study on liquid-liquid bimetal composite casting hammers," China Foundry, vol. 11, 2014, pp. 412-417.
[4] L Sachin, Nimbulkara, and S Rajendra, "Design optimization of gating and feeding system through simulation technique for sand casting of wear plate," Perspectives in Science, vol. 8, 2016, pp. 3942.

[5] H. Liang, P. Z. Xie, and C. K. Wu, "Research on continuous corefilling casting forming process of copper-clad aluminum bimetal composite material," Acta Metall. Sin.(Engl. Lett.), vol.23, 2010, pp. 206-214.

[6] G. R. Xiong, M. Z. Zheng, and L. Z. Zhao, "Research state on technology of metal matrix composites prepared by casting process," Casting Technology, vol. 4, 2006, pp. 563-565.

[7] Y. C. Zhu, Z. J. Wei, and S. F. Rong, "Analysis on the formation mechanism of bimetal composite layer between LAS and HCCI," China Foundry, vol. 13, 2016, pp. 396-401.

[8] S. F. Rong and Y. C. Zhu, "Study on lining board with bimetal liquid composite casting," Advanced Materials Research, vol. 317, 2011, pp. $158-161$.

[9] Y. C. Zhu, Z. J. Wei, and S. F. Rong, "Analysis of heat transport and thickness of bimetal composition layer between low-alloy steel and high-chromium white cast iron," Materials Research Innovations, vol. 19, 2016, pp. 561-565.

[10] C. Joseph. Benedyk, "Novelis Fusion Process-Breakthough in the simultaneous DC casting of multiple aluminum alloy layers for rolling ingot," LIGHT METAL AGE, vol. 8, 2006, pp. 48-50.

[11] E. Takeuchi, M. Zeze, and H. Tanaka, "Mizoguchi. Novel continuous casting process for clad steel slabs with level DC magnetic field. Ironmaking and Steelmaking, vol. 24, 1997, pp. 257-261.

[12] R. Zareie and H. R. Akbari Mousavi, "The effect of explosive welding parameters on metallurgical and mechanical interfacial features of Inconel 625/plain carbon steel bimetal plate," Materials Science \& Engineering A," vol. 556, 2012, pp. 454-463.

[13] Li Qingchun, Basis of Cast Forming Theory, Beijing: Mechanical Industry Press, 1982, pp. 127. 